\title{
On the Connection between Planets, Dark Matter and Cancer: Comment on "Planetary Dependence of Melanoma"
}

\author{
Hector Socas-Navarro \\ Instituto de Astrofísica de Canarias \\ Avda Vía Láctea S/N, La Laguna 38205 \\ Tenerife 38205, Spain \\ Departamento de Astrofísica \\ Universidad de La Laguna \\ La Laguna, Tenerife 38205, Spain \\ hsocas@iac.es
}

Received 3 December 2018

Accepted 10 December 2018

Published 10 January 2019

\begin{abstract}
In a recent paper, Zioutas and Valachovic (2018) claim that dark matter is responsible for a significant fraction of the melanoma skin cancer. This conclusion is drawn from their observation of a significant correlation between skin melanoma incidence in the US and the inner planets positions (especially those of Mercury and Earth). Here, I present a number of objections to their interpretation. Some (but not all) of the counterarguments are based on the analysis of a larger dataset from the same source, considering more cancer types and separating by patient attributes, such as race. One of the counterarguments is that if the melanoma fluctuations with periods similar to planetary orbits were produced by dark matter density enhancements on Earth, then we would have to conclude that the black population is somehow immune to dark matter, a conclusion that seems incompatible with the current Weakly-Interacting Massive Particles (WIMP) paradigm. Interestingly, some periodicities are present in the data, including the ones reported by Zioutas and Valachovic, but I argue that they must have a societal rather than astronomical origin.
\end{abstract}

Keywords: Cancer; melanoma; planetary correlations; dark matter.

\section{Introduction}

In the most widely accepted cosmological model (the $\Lambda$ CDM cosmology, named after Einstein's cosmological constant $\Lambda$ and the Cold Dark Matter acronym), dark matter is an invisible substance that accounts for nearly $80 \%$ of all matter in the Universe. ${ }^{7}$ It is not affected by electro-magnetic forces and, as such, cannot be seen or touched, which has allowed this enigmatic form of matter to elude direct detection thus far.

This is an Open Access article published by World Scientific Publishing Company. It is distributed under the terms of the Creative Commons Attribution 4.0 (CC-BY) License. Further distribution of this work is permitted, provided the original work is properly cited. 
A vast array of astrophysical observations during the last decades has resulted in strong constraints on its properties, leading to a majority consensus among experts in favor of the Weakly-Interacting Massive Particles (WIMP) scenario. ${ }^{10}$ Basically, dark matter would be a fluid of subatomic particles interacting only by gravity and the weak nuclear force. In this respect, the dark matter particles would be similar to neutrinos, except that neutrinos move at nearly the speed of light whereas the dark matter particles inferred by galactic and cosmological observations must be moving at much lower, non-relativistic speeds (this is the meaning of "cold" in the CDM acronym). The dark matter fluid should be in the form of nearly spherical halos, in which galaxies are embedded. In our Milky Way, dark matter particles in the solar neighborhood should be moving at speeds of the order of $230 \mathrm{~km} / \mathrm{s}$. Being electrically neutral, dark matter particles do not come together to form molecules and do not collapse to form disks, stars or planets, like ordinary matter does. In the ideal case of an isolated galaxy, a dark matter halo would have a smooth, spherically symmetric density distribution, without structure at scales much smaller than the galaxy size. ${ }^{4}$ However, tidal interactions or gravitational perturbations from nearby objects may induce substructure in the galactic halos, such as the so-called streams. While observational evidence of such substructures has not yet been found, it is being actively sought by astronomers.

Because dark matter has a rather uniform distribution at interstellar scales, it has a relatively low density compared to ordinary matter in the vicinity of stars. This is because ordinary matter is extremely concentrated in and around stars, separated by colossal distances of empty space between them. The amount of dark matter mass contained in the solar system is estimated to be comparable to that of a large asteroid. This is the reason why dark matter is only important at galactic scales or larger.

Every second, a number of dark matter particles, moving at speeds of hundreds of $\mathrm{km} / \mathrm{s}$, pass through our bodies. The actual number depends on the WIMP mass (or mass distribution), which is unknown. Since these particles could, in principle, collide and interact with the atomic nuclei in our cells, they might be able to alter the DNA composition and induce mutations in our genes, thus increasing the risk for developing cancerous tumors. Given our present lack of knowledge on the WIMP properties, it is not possible to estimate the rates of collisions or mutations produced by these particles. ${ }^{1-13}$ However, as the solar system moves through the galaxy, it is expected to encounter different dark matter densities on scales from tens to hundreds of millions of years. Some authors have sought indirect evidence of dark matter interaction fluctuations in the geological or fossil records. ${ }^{8,9}$ Connections between cancer rate variations and dark matter density have been proposed in the literature.

Zioutas and Valachovic ${ }^{14}$ (hereafter ZV18) claimed to have found a link between periodic variations of skin melanoma and dark matter density changes driven by planetary motions in the solar system. Their paper is not the first to put forward a causal relation between dark matter and cancer, but it is the first to correlate it with 
planetary motions and on timescales of months, as opposed to at least tens of millions of years in previous works.

In the spirit of healthy scientific debate, this paper puts forward some objections to the conclusions of ZV18 that should probably be kept in mind in future studies on the issue which, undoubtedly, is an important one. As they point out, cancer and the "Dark Universe" are among the biggest mysteries in medicine and physics, respectively. The following sections present first an introduction to the cancer data analyzed here and then the counterarguments to the planetary link hypothesis in ascending order of strength.

\section{Cancer Database}

ZV18 employed for their analysis the incidence of skin melanoma compiled in the database SEER9 from the Surveillance Epidemiology End Results of the US National Cancer Institute. This dataset comprises records of individual patients diagnosed between 1973 and 2015 in a sample population from Atlanta, Connecticut, Detroit, Hawaii, Iowa, New Mexico, San Francisco-Oakland, Seattle-Puget Sound and Utah. The database is organized by eight major cancer categories (breast, colon and rectum, digestive, female genital, male genital, lymphoma and leukemia, respiratory and urinary) and a miscellaneous category that includes several other types, including skin melanoma. The data presented in this paper are from the same database, although some results discussed below have been obtained from the analysis of other types of cancer.

\section{Counterarguments to the Planetary Link}

\subsection{Dark matter structure}

The astrophysical scenario considered by ZV18 is not described in their paper. They assume the presence of a dark matter stream running through the solar system. The correlation between planet positions and skin melanoma is interpreted as a result of the planets periodically increasing the density of dark matter passing through Earth. However, this overall picture is very difficult, if not impossible, to translate into a detailed layout. Consider Mercury, for instance. Let us assume that there is a dark matter stream and Mercury moves into and out of the stream regularly during its orbit. Furthermore, in order to produce the observed periodicity, this geometry (and the position at which Mercury dives into the stream) must remain the same for the entire period of 38 years analyzed by ZV18. The Sun revolves around the galaxy at approximately $200 \mathrm{~km} / \mathrm{s}$, which means that it has traveled $2.4 \times 10^{12} \mathrm{~km}$ in 38 years. In contrast, Mercury's orbit is only 58 million $\mathrm{km}$. This mismatch of several orders of magnitude between both distances makes it nearly impossible to construct a suitable geometrical scenario unless the dark matter stream is perfectly aligned with the solar system motion through the galaxy to a precision better than $10^{-4}$ radians (a percent of a degree). 


\subsection{Relative planet positions}

Even if Mercury and Venus were periodically diving into a stream, focusing or splashing dark matter around, the position of Earth around its orbit would be different each time they do this. In other words, for Mercury to produce repeated enhancements of dark matter density at Earth, one would need to replicate the relative positions of Mercury, Earth and the stream. We would not have a periodicity with Mercury's orbital period because every time Mercury encounters the dark matter stream, Earth would be at different location.

\subsection{Diagnosis delay}

The dates recorded in the SEER database reflect the times when the cancer was diagnosed, not when the patients became sick, much less when they were exposed to a possible causing carcinogenic agent. Cancer diagnosis delay is an extremely important parameter in medicine, given its influence on the survival rate, and numerous studies exist in the scientific literature. Such studies usually consider the time from the patient noticing the first symptoms to consultation with a primary care physician and then the further delay to be referred and diagnosed by a specialist. Existing studies for the particular case of skin melanoma conclude that the delay from symptoms to diagnosis is typically of the order of 11 months $^{3}$ or, more recently, 7 months. ${ }^{12}$ In the context of timing a possible dark matter excess as a potential carcinogen, one would need to consider an additional term, namely the delay from exposure to the appearance of the first symptoms which, to the author's knowledge, has not been quantified. Diagnosis delay is then an average of 2 to 4 times longer than Mercury's orbit, which means that, even if there was a causal connection between Mercury's position and the incidence of melanoma, it would be completely washed out in the data by the effect of diagnosis delay. A similar statement could be made for Venus or Earth, with somewhat lower ratios of delay to claimed periodicity, down to between 0.6 and 0.9 for the case of Earth.

\subsection{Periodicities}

Although ZV18 restricted their analysis to the case of skin melanoma, the SEER database includes many other types of cancer. Figure 1 shows the Fourier power spectrum of eight major categories in the database alongside the skin melanoma, which is displayed separately for white and black populations.

The power spectrum exhibits a number of conspicuous peaks above the noise, indicative of periodicities in the rate of cancer diagnosis. The most prominent peak occurs at one year. A simple explanation for this periodicity is that it is caused by human habits such as routine medical examinations, which are typically scheduled on a yearly basis. The other peaks at integer positions are easily explained as harmonics of the one-year peak. The Fourier decomposition of a simple harmonic function (a sine or cosine signal) results in a single peak at the signal frequency. 


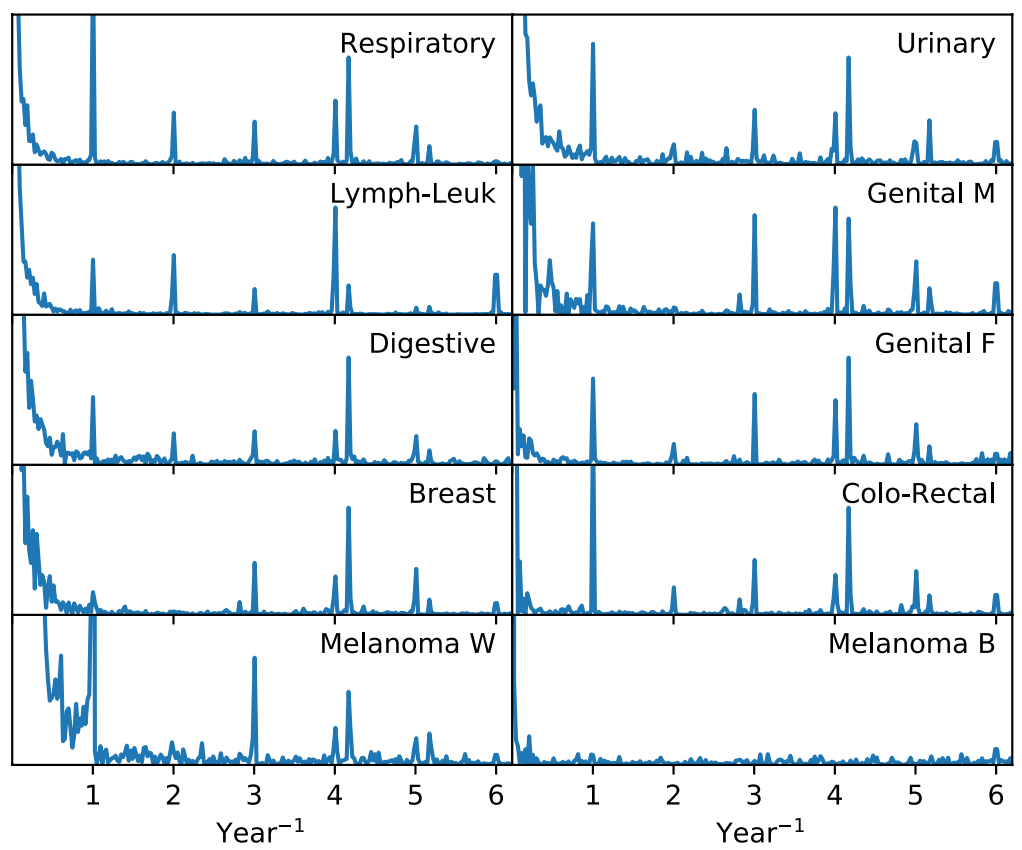

Fig. 1. Fourier power spectra of eight major cancer categories in the SEER9 database, along with the skin melanoma for white and black populations. Incidence is per month (normalized to 30.44 days) and 100,000 population. The "Lymph-Leuk" label refers to "lymphoma and leukemia". "Genital $M$ " and "Genital F" refers to male and female genital cancer, respectively.

However, if we have a function that is periodic but not sinusoidal, we obtain a peak at the fundamental frequency plus a number of other peaks at the multiples of that frequency (harmonics). The relative amplitudes of the harmonics depend on the specific shape of the function. Therefore, all the peaks at integer abscissae values in Fig. 1 may be ascribed to a yearly variation in cancer diagnosis rate, which is more frequent in the summer of the northern hemisphere. ${ }^{\text {a }}$

Two peaks in the power spectra remain unaccounted. Their frequencies and widths are $4.17 \pm 0.01$ and $5.17 \pm 0.01$ year $^{-1}$, respectively. ZV18 associated the first one with Mercury's orbit, which is of 88 days or 4.15 year $^{-1}$. They do not give any explanation for the peak at 5.17. The analysis presented here does not show any feature with the frequency of Venus orbit at 1.62 years $^{-1}$ (225 days).

Figure 1 shows that all the main cancer categories in the SEER database exhibit the same periodicities, the peaks are located at the same frequency positions. However, the ZV18 paper presents a connection of dark matter to skin melanoma only. The reasons for this are not clear. They do not mention whether they analyzed the other data series or what was the motivation to focus on skin melanoma in particular. 


\subsection{Race immunity}

An interesting observation from Fig. 1 is the absence of skin melanoma among the black population (bottom-right panel). Skin pigmentation is a well-known protective factor against ultraviolet radiation and skin cancer development. However, there is no known reason why it should protect against dark matter. In order to explore this possibility, we need to isolate the contribution of dark matter, separating it from the rest of the white population melanoma incidence. If, as claimed by ZV18, the 4.17 year $^{-1}$ peak is due to dark matter density fluctuations induced by Mercury, we can filter out that frequency and analyze the two contributions separately. The filtered signal (first component) would be the cancer incidence due to normal (non-dark matter-related) causes and may be different for different races. The remaining (second component) would account for those cases that have been induced by dark matter. A simple filtering would give us a fluctuating second component that oscillates between a negative and a positive value, corresponding to the hypothetical fluctuation in cases due to dark matter. We are interested in the amplitude of this fluctuation.

Figure 2 (blue) shows the second component envelope as a function of time, i.e., the variation of the skin melanoma incidence in the white population supposedly induced by dark matter. At the very least, the total incidence of skin melanoma in the black population should have a comparable amplitude to the blue curve. However, Fig. 2 shows that the total incidence for the black population (orange) is always much smaller than the second component in the white population. This means that, in the ZV18 hypothesis (that Mercury's orbit is periodically enhancing the incidence

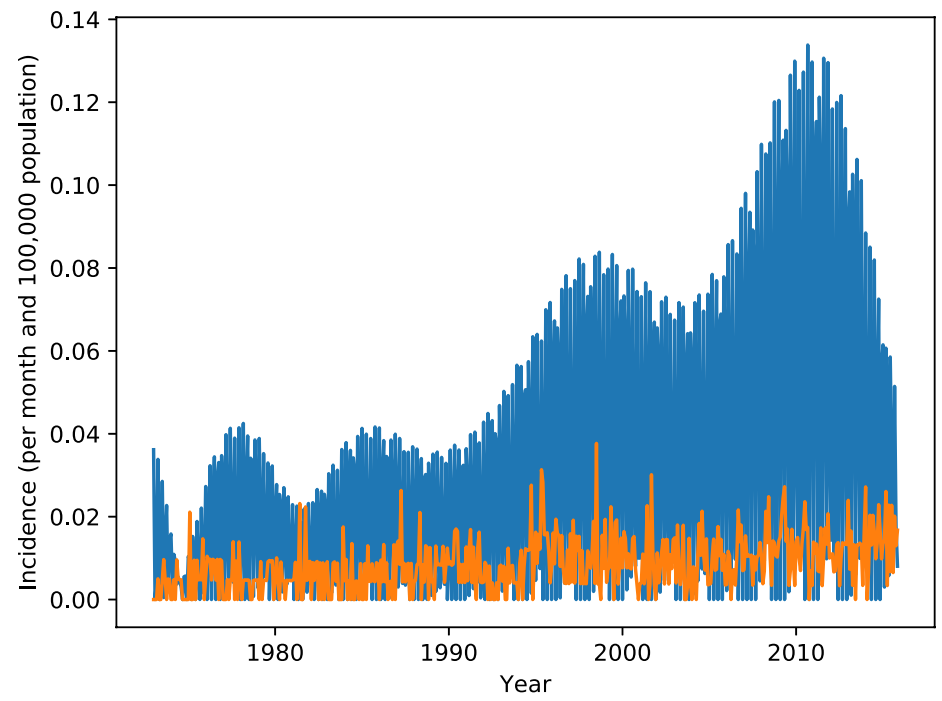

Fig. 2. (Color online) Blue: Time variation of melanoma skin cancer incidence associated to the 4.17 year $^{-1}$ peak in the white population. Orange: Total cancer incidence for the black population. Note that both curves are normalized by time and population. 
of skin melanoma with an 88-day period), this enhancement does not affect the black population, which would be difficult to explain in a model where dark matter does not have electro-magnetic interaction (by definition) and can only interact with atomic nuclei.

\section{Conclusions}

The paper of ZV18 put forward a very bold claim which, if true, would have a tremendous impact on physics and physiology, namely that there is a causal connection between dark matter, planetary motions and melanoma skin cancer. In particular, they highlight a link with the motion of Mercury, which is by far the clearest signal according to their analysis. This work presents a number of objections to that claim. It does not rule out a possible relationship between dark matter and cancer but, if such relationship exists, it cannot be probably derived from these data.

It is possible that dark matter might form structures of galactic scale and, in fact, we have identified streams of stars where wisps of dark matter probably exist. Furthermore, it is possible that the solar system moves through such structures, probing a varying dark matter density. The most obvious example is the $\mathrm{S} 1$ stream in which the solar system is actually embedded. ${ }^{5}$ However, the time scales involved in such transits are much longer. For instance, the Sun has been moving through the S1 stream for roughly 5 million years and will continue to do so for a similar amount of time. Another dark matter structure that has been hypothesized is a galactic disk, assuming that dark matter exhibits some (extremely weak) degree of interaction with itself. The oscillatory motion of the solar system across the galactic plane would make it plunge into and out of such disk but that would occur on periods of about 60 million years. However, on planetary motion scales, and particularly on scales of less than three months, it is not expected to find such variations. There is no plausible astrophysical scenario that would produce a splash of dark matter as Mercury moves in and out of it consistently for over 40 years. This is exacerbated if we consider that the relative positions of Mercury and Earth are changing continuously. There is no way to reproduce a geometry between Earth, Mercury and the putative dark matter source with the periodicity of Mercury's orbit.

Even if we forget about all the above considerations, there is a very important problem with the ZV18 interpretation. The data that we have do not reflect the time when the disease was contracted or even when it manifests its first symptoms. We have the date of diagnosis. Diagnosis delay is an important problem in medicine and it would smear out any possible periodic signal that could have existed, such as the putative planetary connection. The causes for any periodicities in the data should be sought first in how we approach the search and discovery of cancer in patients. The one-year period and its harmonics found in most types of cancer are probably a direct consequence of our medical examination habits. Interestingly, there are two periodicities that, to the author's best knowledge, remain unexplained. These peaks are located at 4.17 and 5.17 year $^{-1}$ (87 and 70 days). 
Even if we forget about astrophysics and we also forget about diagnosis delay, the data show that the black population is unaffected by the dark matter-induced modulation that ZV18 claim to see in the overall incidence of skin melanoma. Skin pigmentation protects against the harmful effects of ultraviolet radiation and it is less prone to cancer development but there is no known reason why a darker skin should make people immune to dark matter.

From all the above considerations, it appears that the link between Mercury's orbital period and a periodicity in cancer (of many types) diagnosis is casual and not causal. No evidence is observed in this analysis for a similar coincidence with Venus.

\section{Acknowledgments}

The author gratefully acknowledges the financial support from the Spanish Ministry of Economy and Competitivity through project AYA2014-60476-P. This research has made use of NASA's Astrophysics Data System Bibliographic Services. The Python Matplotlib, ${ }^{2}$ Numpy $^{11}$ and iPython ${ }^{6}$ modules have been employed to generate the figures and calculations in this paper.

\section{References}

1. K. Freese and C. Savage, Phys. Lett. B 717, 25-28 (2012).

2. J. D. Hunter, Comput. Sci. Eng. 9(3), 90-95 (2007).

3. J. Krige, S. Isaacs, D. Hudson, H. S. King, R. M. Strover and C. Johnson, Cancer, 68(12), 2064-2068 (1991).

4. J. F. Navarro, C. S. Frenk and S. D. M. White, Astrophys. J. 462, 563 (1996).

5. C. A. J. O'Hare, C. McCabe, N. W. Evans, G. Myeong and V. Belokurov, Phys. Rev. Lett. D 98, 103006 (2018).

6. F. Pérez and B. E. Granger, Comput. Sci. Eng. 9(3), 21-29 (2007).

7. Planck Collab. (N. Aghanim, Y. Akrami, M. Ashdown, J. Aumont, C. Baccigalupi, M. Ballardini, A. J. Banday, R. B. Barreiro, N. Bartolo, S. Basak, R. Battye, K. Benabed, J.-P. Bernard, M. Bersanelli, P. Bielewicz, J. J. Bock, J. R. Bond, J. Borrill, F. R. Bouchet, F. Boulanger, M. Bucher, C. Burigana, R. C. Butler, E. Calabrese, J.-F. Cardoso, J. Carron, A. Challinor, H. C. Chiang, J. Chluba, L. P. L. Colombo, C. Combet, D. Contreras, B. P. Crill, F. Cuttaia, P. de Bernardis, G. de Zotti, J. Delabrouille, J.-M. Delouis, E. Di Valentino, J. M. Diego, O. Doré, M. Douspis, A. Ducout, X. Dupac, S. Dusini, G. Efstathiou, F. Elsner, T. A. Enßlin, H. K. Eriksen, Y. Fantaye, M. Farhang, J. Fergusson, R. Fernandez-Cobos, F. Finelli, F. Forastieri, M. Frailis, E. Franceschi, A. Frolov, S. Galeotta, S. Galli, K. Ganga, R. T. Génova-Santos, M. Gerbino, T. Ghosh, J. González-Nuevo, K. M. Górski, S. Gratton, A. Gruppuso, J. E. Gudmundsson, J. Hamann, W. Handley, D. Herranz, E. Hivon, Z. Huang, A. H. Jaffe, W. C. Jones, A. Karakci, E. Keihänen, R. Keskitalo, K. Kiiveri, J. Kim, T. S. Kisner, L. Knox, N. Krachmalnicoff, M. Kunz, H. Kurki-Suonio, G. Lagache, J.-M. Lamarre, A. Lasenby, M. Lattanzi, C. R. Lawrence, M. Le Jeune, P. Lemos, J. Lesgourgues, F. Levrier, A. Lewis, M. Liguori, P. B. Lilje, M. Lilley, V. Lindholm, M. López-Caniego, P. M. Lubin, Y.-Z. Ma, J. F. Macías-Pérez, G. Maggio, D. Maino, N. Mandolesi, A. Mangilli, A. Marcos-Caballero, M. Maris, P. G. Martin, M. Martinelli, E. Martínez-González, S. Matarrese, N. Mauri, J. D. McEwen, P. R. Meinhold, A. Melchiorri, A. Mennella, M. Migliaccio, 
M. Millea, S. Mitra, M.-A. Miville-Deschênes, D. Molinari, L. Montier, G. Morgante, A. Moss, P. Natoli, H. U. N ørgaard-Nielsen, L. Pagano, D. Paoletti, B. Partridge, G. Patanchon, H. V. Peiris, F. Perrotta, V. Pettorino, F. Piacentini, L. Polastri, G. Polenta, J.-L. Puget, J. P. Rachen, M. Reinecke, M. Remazeilles, A. Renzi, G. Rocha, C. Rosset, G. Roudier, J. A. Rubi ñ o-Mart ín, B. Ruiz-Granados, L. Salvati, M. Sandri, M. Savelainen, D. Scott, E. P. S. Shellard, C. Sirignano, G. Sirri, L. D. Spencer, R. Sunyaev, A.-S. Suur-Uski, J. A. Tauber, D. Tavagnacco, M. Tenti, L. Toffolatti, M. Tomasi, T. Trombetti, L. Valenziano, J. Valiviita, B. Van Tent, L. Vibert, P. Vielva, F. Villa, N. Vittorio, B. D. Wandelt, I. K. Wehus, M. White, S. D. M. White, A. Zacchei and A. Zonca). arXiv:1807.06209 [astro-ph.co].

8. M. R. Rampino, Mon. Not. R. Astron. Soc. 448, 1816-1820 (2015).

9. L. Randall, Dark Matter and the Dinosaurs: The Astounding Interconnectedness of the Universe (Harper Collins, New York City, Random House, 2016).

10. L. Roszkowski, E. M. Sessolo and S. Trojanowski, Rep. Prog. Phys. 81(6), 066201 (2018).

11. S. Van Der Walt, S. C. Colbert and G. Varoquaux, Comput. Sci. Eng. 13(2), 22-30 (2011).

12. M. H. S. B. Xavier, A. P. Drummond-Lage, C. Baeta, L. Rocha, A. M. Almeida and A. J. A. Wainstein, Medicine 95, e4396 (2016).

13. K. Zioutas, Phys. Lett. B 242, 257-264 (1990).

14. K. Zioutas and E. Valachovic, Biophys. Rev. Lett. 13, 75-92 (2018). 